\title{
Interference Mitigation under Degrees-of-Freedom Sensing Uncertainties in Opportunistic Transmission
}

\author{
Jordi Borràs and Gregori Vazquez \\ Department of Signal Theory and Communications, Technical University of Catalonia (UPC) \\ Building D5, Campus Nord UPC, Jordi Girona 1-3, 08034 Barcelona, Spain \\ Email: \{jordi.borras.pino, gregori.vazquez\}@upc.edu
}

\begin{abstract}
Inter-system interference may limit the performance of co-existing systems in dense heterogeneous wireless networks. Context-aware waveform design can profitably overcome this limitation. However, the latter substantially depends on degreesof-freedom (DoF) sensing mechanisms. In this work, we show that total least-squares (TLS)-based waveform design is robust against sensing uncertainties. Given the equivalence of minimum norm and TLS, the latter exhibits the good properties of linear predictors, which are of paramount importance to guarantee minimum inter-system interference and detectability by neighboring nodes. Additionally, since derived solution relies on orthogonal projector onto the so-called noise subspace, we can efficiently and iteratively construct an orthogonal waveform-book enabling the presented transmission scheme in multi-carrier scenarios. Simulation results are presented to support our theoretical contributions, and to highlight any potential advantage of proposed solution in crowded heterogenous wireless networks.
\end{abstract}

Index Terms-Opportunistic communications, distributed networks, total least-squares, sensing uncertainties, waveform design.

\section{INTRODUCTION}

Next-era wireless communications will be mainly characterized by demanding high data-rates, ultra low latency, high reliability, and a huge amount of wireless interfaces simultaneously transmitting information [1]. Moreover, another important consideration is the co-existence of several communication technologies. Therefore, many works recently presented study the communication in such heterogeneous environments with different technologies co-existing. Nevertheless, interference might pose a severe limitation in dense wireless networks. Hence, several recent works deal with techniques for interference management, avoidance, and/or cancellation (see [2-4] and references therein).

Opportunistic communication [5] has arisen as a promising technique which efficiently and robustly exploits network resources. This communication format has a context- or scenario-aware nature. Therefore, it is suitable to be employed in decentralized or infrastructureless networks [6]. Since inefficient backbone communication can be avoided, latency might be reduced. Furthermore, since opportunistic transmissions are adapted to network conditions, transmission schemes theirselves may also present interference management capabilities. Some examples of opportunistic communications techniques

This work has been supported by the Spanish Ministry of Science, Innovation and Universities through project WINTER: TEC2016-76409-C2-1-R (AEI/FEDER, UE) and fellowship FPI BES-2017-080071, and by the Catalan Government (AGAUR) under grant 2017 SGR 578. are heterogeneous networks [7], interweave cognitive radio [8], and device-to-device communications [9].

In the following, we detail some recent results in the context of opportunistic communications. Although waveform design has been widely studied (see, e.g., [10-12] and references therein), it is still an open issue. In [13], a waveform design scheme which permits the co-existence of radar and mobile systems is proposed. Additionally, [14] addresses the performance analysis of improper Gaussian signaling in interweave cognitive radio systems. In view of the expected lack of radio resources to satisfy the demanding user-requirements of a large number of inter-connected devices, in [15] we may find a survey of state-of-the-art proposals of non-orthogonal multiple-access (NOMA) for cognitive communications.

What is very interesting is the marriage of opportunistic communications and novel proposals for increase the per-user rate. For instance, opportunistic communications have been recently studied to operate in millimeter-wave bands [16], and altogether with full-duplex technology [17].

As previously presented, interference might be a limiting performance factor in distributed wireless networks. Therefore, some state-of-the-art proposals are also concerned with interference management (see [18] and references therein).

As a final remark on the literature, we focus on performance analysis. For instance, the impact of sensing performance in detection error in cognitive systems is assesed in [19]. Regarding to the throughput, [20] studies the achievable rates of interweave cognitive radio, whereas a study of the optimal throughput in multi-cell opportunistic multiple-access can be found in [21].

In this work, we will study the problem of robust waveform design. Actually, according to our previous results [22-24], minimum-norm waveform optimization has found to be the best that can be done under degrees-of-freedom (DoF) uncertainties in a per-local only sensing scheme. In this paper, we tackle the problem in a different manner which permits a total least-squares (TLS) formulation.

The remainder of this paper is organized as follows. In Sec. II we present the considered signal model and formulate the problem in detail. The robust waveform design is addressed Sec. III. As well, we compare the result with previous works and propose a multi-waveform solution. Some properties of the presented solution are detailed in Sec. IV. Simulation results are reported in Sec. V and the paper is concluded in Sec. VI. 


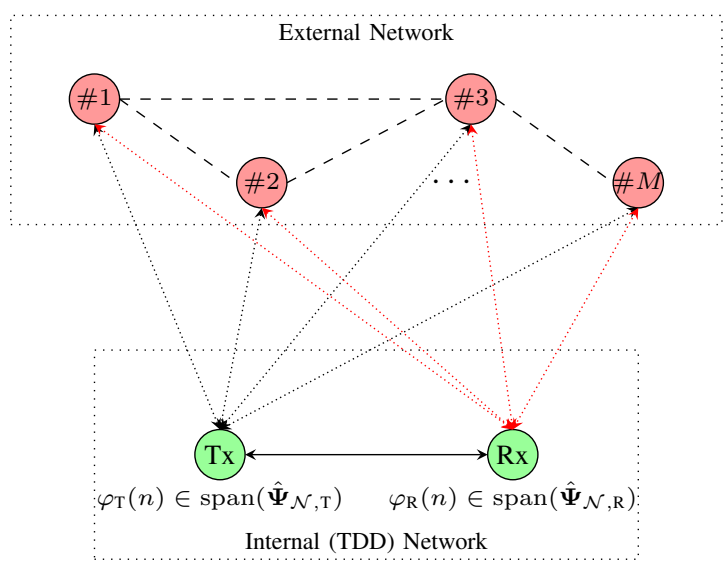

Fig. 1: Context-aware opportunistic transmission in a distributed network.

\section{Signal Description AND Problem Formulation}

In the sequel, we consider the scenario shown in Fig. 1. External network refers to the wireless environment, consisting in an arbitrary number $M$ of wireless interfaces simultaneously exploiting a fraction of the total degrees-of-freedom (DoF), $N$, (cf. [25], [26]). Internal network consists in those users wishing to opportunistically exploit the available DoF. For the sake of simplicity, we consider herein that it is composed of a transmitter-receiver pair. To that end, internal-network users sense the network state through interference channels, and decide, by solving a binary hypothesis testing problem, whether a $\operatorname{DoF} \zeta_{n}$, for $n=1, \ldots, N$, is occupied or available:

$$
\begin{aligned}
& \mathcal{H}_{0}: \zeta_{n} \text { is available, } \\
& \mathcal{H}_{1}: \zeta_{n} \text { is occupied. }
\end{aligned}
$$

This problem involves the use of an arbitrary sensing mechanism. Spectrum sensing, or more general DoF sensing, has been widely studied in the literature (cf. [27] and references therein). Hence, the sensing stage is assumed to be completed in this work. The sensing mechanism chosen by each internalnetwork user allows it to construct a basis of the occupied DoF subspace, usually known as signal subspace, and a basis of the available DoF subspace, also known as noise subspace. Once these bases are obtained at each internalnetwork node, they have to design, without side information on the subspaces sensed at neighboring nodes, a waveform or shaping filter $\varphi_{\mathrm{i}}(n)$, for $\mathrm{i}=\{\mathrm{T}, \mathrm{R}\}$, to set up the opportunistic communication. By letting $a[m], T$ and $S_{\mathrm{R}}$ be a symbol from a given constellation, the symbol period and the received power, the signal observed by internal-network receiver is given by

$$
y(n)=\sum_{m} \sqrt{S_{\mathrm{R}}} a[m] \varphi_{\mathrm{T}}(n-m T)+v(n),
$$

where $v(n) \sim \mathcal{C N}\left(0, \sigma^{2}\right)$ is a complex noise. Then, internal receiver will use its local waveform $\varphi_{\mathrm{R}}(n)$ to detect $y(n)$, without cooperating with internal transmitter. This challenging issue is addressed by the authors in [24]. In this work, we will focus on designing robust waveforms in the sense of minimum inter-system interference.

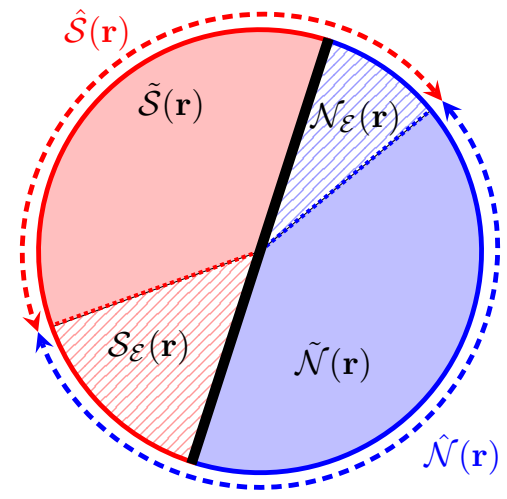

Fig. 2: Sketch of signal and noise subspaces at geographical position $\mathbf{r}$.

\section{A. Degrees-of-Freedom Detection and Sensing Uncertainties}

Although sensing schemes are being continuously improved (see, e.g., [28]), since they are based on detection theory, inherent errors due to false-alarm and miss-detection probabilities are always committed yielding sensing uncertainties.

Notice that there is a slight abuse of notation when we define the external network. Actually, each internal-network node may observe different external networks, depending on their geographical position space coordinates $\mathbf{r} \triangleq[x, y, z]^{T}$. Therefore, we will focus our discussion on an arbitrary $\mathbf{r}$. According to Fig. 2, signal and noise subspaces, of dimensions $D(\mathbf{r})$ and $K(\mathbf{r})$, respectively, can be written as $\mathcal{S}(\mathbf{r})=$ $\tilde{\mathcal{S}}(\mathbf{r}) \cup \mathcal{S}_{\mathcal{E}}(\mathbf{r})$ and $\mathcal{N}(\mathbf{r})=\tilde{\mathcal{N}}(\mathbf{r}) \cup \mathcal{N}_{\mathcal{E}}(\mathbf{r})$. In this work, we assume that $\tilde{\mathcal{S}}(\mathbf{r})$ and $\mathcal{S}_{\mathcal{E}}(\mathbf{r})$ contain the occupied DoF sensed as occupied and the occupied DoF erroneously sensed as available, respectively. Regarding to $\mathcal{N}(\mathbf{r})$, we refer to $\tilde{\mathcal{N}}(\mathbf{r})$ and $\mathcal{N}_{\mathcal{E}}(\mathbf{r})$ as the available DoF detected as available and the available DoF erroneously detected as occupied, respectively. Therefore, signal and noise subspaces bases are given by

$$
\begin{aligned}
\boldsymbol{\Psi}_{\mathcal{S}}(\mathbf{r}) & =\left[\begin{array}{l:l}
\tilde{\boldsymbol{\Psi}}_{\mathcal{S}}(\mathbf{r}) & \boldsymbol{\Xi}(\mathbf{r})
\end{array}\right], \\
\boldsymbol{\Psi}_{\mathcal{N}}(\mathbf{r}) & =\left[\begin{array}{lll}
\tilde{\mathbf{\Psi}}_{\mathcal{N}}(\mathbf{r}) & \mathbf{\Upsilon}(\mathbf{r})
\end{array}\right],
\end{aligned}
$$

with $\tilde{\mathcal{S}}(\mathbf{r})=\operatorname{span}\left(\tilde{\boldsymbol{\Psi}}_{\mathcal{S}}(\mathbf{r})\right), \mathcal{S}_{\mathcal{E}}(\mathbf{r})=\operatorname{span}(\boldsymbol{\Xi}(\mathbf{r})), \tilde{\mathcal{N}}(\mathbf{r})=$ $\operatorname{span}\left(\tilde{\boldsymbol{\Psi}}_{\mathcal{N}}(\mathbf{r})\right)$, and $\mathcal{N}_{\mathcal{E}}(\mathbf{r})=\operatorname{span}(\boldsymbol{\Upsilon}(\mathbf{r}))$. Taking the latter into account, we can define the sensed bases as

$$
\begin{aligned}
\hat{\mathbf{\Psi}}_{\mathcal{S}}(\mathbf{r}) & =\left[\begin{array}{l:l}
\tilde{\mathbf{\Psi}}_{\mathcal{S}}(\mathbf{r}) & \mathbf{\Upsilon}(\mathbf{r})
\end{array}\right], \\
\hat{\mathbf{\Psi}}_{\mathcal{N}}(\mathbf{r}) & =\left[\begin{array}{ll:}
\tilde{\boldsymbol{\Psi}}_{\mathcal{N}}(\mathbf{r}) & \boldsymbol{\Xi}(\mathbf{r})
\end{array}\right],
\end{aligned}
$$

with dimensions $\hat{D}(\mathbf{r})=D(\mathbf{r})-\xi(\mathbf{r})+(K(\mathbf{r})-\tilde{K}(\mathbf{r}))$ and $\hat{K}(\mathbf{r})=\tilde{K}(\mathbf{r})+\xi(\mathbf{r})$, respectively, being $\xi(\mathbf{r})=\operatorname{dim}\left[\mathcal{S}_{\mathcal{E}}(\mathbf{r})\right]$ and $\tilde{K}(\mathbf{r})=\operatorname{dim}[\tilde{\mathcal{N}}(\mathbf{r})]$. From the transmission point of view, note that false-alarm rate only supposes that some transmission opportunities are lost, which is not critical in view of intersystem interferences. Nevertheless, miss detection may lead to these undesired interferences, since internal-network node at coordinates $\mathbf{r}$ will exploit the DoF encompassed in $\boldsymbol{\Xi}(\mathbf{r})$. As a final remark, if an occupied DoF is sensed as available due to poor monitoring conditions (outage or shadowing and multipath fadings), no interferences will be delivered to external system due to time division duplex (TDD) assumption. 


\section{Robust Signaling BASED ON A TotaL LEAST-SQUARES OPTIMIZATION}

In this Section, we present the main result of this work. As explained before, since we address the design at an arbitrary geographical position, we drop the dependence on the position vector $\mathbf{r}$ for simplicity of notation whenever it is possible.

It is worth noting that any waveform $\varphi$ fulfilling

$$
\boldsymbol{\Psi}_{\mathcal{S}}^{H} \boldsymbol{\varphi}=\mathbf{0}
$$

will not provide inter-system interference. However, as we have discussed, we will not be able to sense an actual signal subspace basis. Therefore, we will consider herein the worst case, i.e. we seek a waveform $\varphi$ orthogonal to $\hat{\boldsymbol{\Psi}}_{\mathcal{S}}$ and with minimum impact on those DoF encompassed in $\Xi$. To this end, let us define the extended signal subspace basis as follows:

$$
\breve{\Psi}_{\mathcal{S}}=\left[\begin{array}{l:l}
\hat{\boldsymbol{\Psi}}_{\mathcal{S}} & \boldsymbol{\Xi}
\end{array}\right]
$$

Thus, the problem reduces to find a waveform $\varphi$ such that

$$
\left[\begin{array}{l:l}
\hat{\boldsymbol{\Psi}}_{\mathcal{S}} & \boldsymbol{\Xi}
\end{array}\right]^{H} \boldsymbol{\varphi}=\mathbf{0}+\mathbf{e}_{\mathcal{S}}
$$

where $\mathbf{e}_{\mathcal{S}}$ stands for the error with respect to the complete orthogonality. For the sake of convenience, we define the following matrices:

$$
\begin{aligned}
& \hat{\boldsymbol{\Omega}}_{\mathcal{S}}^{H}=\left[\begin{array}{l:l}
\hat{\mathbf{\Psi}}_{\mathcal{S}} & \mathbf{0}_{N \times \xi}
\end{array}\right], \\
& \mathbf{E}_{\mathcal{S}}^{H}=\left[\begin{array}{l}
\mathbf{0}_{N \times \hat{D}} \\
\boldsymbol{\Xi}
\end{array}\right],
\end{aligned}
$$

such that the problem in (9) can be expressed as

$$
\left(\hat{\Omega}_{\mathcal{S}}+\mathbf{E}_{\mathcal{S}}\right) \varphi=0+\mathbf{e}_{\mathcal{S}} .
$$

It is worth noting that (12) admits a total least-squares (TLS) problem formulation [29], where $\mathbf{E}_{\mathcal{S}}$ and $\mathbf{e}_{\mathcal{S}}$ play the roles of errors in data matrix and observations, respectively. By defining the extended error matrix or perturbation matrix as $\boldsymbol{\Delta} \triangleq\left[\begin{array}{l:l}\mathbf{e}_{\mathcal{S}} & \mathbf{E}_{\mathcal{S}}\end{array}\right]$, TLS finds a matrix $\boldsymbol{\Delta}$ such that

$$
\begin{aligned}
& \min _{\left\{\mathbf{E}_{\mathcal{S}}, \mathbf{e}_{\mathcal{S}}\right\}}\|\boldsymbol{\Delta}\|_{\mathrm{F}}^{2} \\
& \text { subject to }\left(\hat{\boldsymbol{\Omega}}_{\mathcal{S}}+\mathbf{E}_{\mathcal{S}}\right) \boldsymbol{\varphi}=\mathbf{0}+\mathbf{e}_{\mathcal{S}} .
\end{aligned}
$$

According to the rationale followed in the literature (see [29], [30]), TLS seeks a solution that belongs to the nullspace of matrix $\mathbf{T}^{H} \mathbf{T}$, being $\mathbf{T}$ the extended data matrix $\mathbf{T} \triangleq\left[\begin{array}{l:l}\mathbf{0} & \hat{\Omega}_{\mathcal{S}}\end{array}\right]$. Therefore, accounting for the singular value decomposition (SVD) of $\mathbf{T}$,

$$
\mathbf{T}=\left[\begin{array}{l:l}
\mathbf{U}_{1} & \mathbf{U}_{2}
\end{array}\right]\left[\begin{array}{cc:c}
\boldsymbol{\Sigma}_{1} & \mathbf{0} & \mathbf{0} \\
\mathbf{0} & \boldsymbol{\Sigma}_{2} &
\end{array}\right]\left[\begin{array}{c}
\mathbf{V}_{1}^{H} \\
\hdashline \mathbf{V}_{2}^{\bar{H}}
\end{array}\right],
$$

where $\mathbf{V}_{1}$ and $\mathbf{V}_{2}$ span the column-space and the null-space of $\mathbf{T}^{H} \mathbf{T}$, respectively, the TLS waveform yields

$$
\varphi_{\mathrm{TLS}}=\frac{\tilde{\mathbf{V}}_{2} \mathbf{c}^{*}}{\mathbf{c}^{H} \mathbf{c}}
$$

where $\mathbf{c}^{T}$ and $\tilde{\mathbf{V}}_{2}$ come from partitioning $\mathbf{V}_{2}$ as

$$
\mathbf{V}_{2}=\left[\begin{array}{c}
\mathbf{c}^{T} \\
\tilde{\mathbf{V}}_{2}
\end{array}\right]
$$

It is worth noting that, whenever the dimensions of the nullspace of $\mathbf{T}^{H} \mathbf{T}$ is larger than one, the TLS problem may lack a unique solution. In that case, the one with minimum norm should be selected. Finally, if several minimum-norm solutions exists, any of them is valid. Consequently, if internal-network nodes do not pre-agree a selection criterion, a detection ambiguity problem arises as in [24].

What is very interesting to notice is that since $\mathbf{T}^{H} \mathbf{T}$ is

$$
\mathbf{T}^{H} \mathbf{T}=\left[\begin{array}{cc}
0 & \mathbf{0}_{1 \times N} \\
\mathbf{0}_{N \times 1} & \hat{\mathbf{\Psi}}_{\mathcal{S}} \hat{\mathbf{\Psi}}_{\mathcal{S}}^{H}
\end{array}\right],
$$

then the proposed design scheme is seeking a solution belonging to the null-space of $\hat{\boldsymbol{\Psi}}_{\mathcal{S}} \hat{\mathbf{\Psi}}_{\mathcal{S}}^{H}$, or, equivalently, to the column-space of $\hat{\boldsymbol{\Psi}}_{\mathcal{N}} \hat{\mathbf{\Psi}}_{\mathcal{N}}^{H}=\mathbf{I}_{N}-\hat{\mathbf{\Psi}}_{\mathcal{S}} \hat{\mathbf{\Psi}}_{\mathcal{S}}^{H}$. By elemental manipulations, it is straightforward to verify that the proposed solution in (15) is just one column of the orthogonal projector onto the sensed noise subspace. This column depends on which row we have selected to divide $\mathbf{V}_{2}$ in (16). Therefore, given the equivalence of minimum norm and TLS [30], the solution proposed by the authors in [22-24], i.e.

$$
\boldsymbol{\varphi}_{n}^{\star}=\left(\mathbf{e}_{n}^{H} \hat{\boldsymbol{\Psi}}_{\mathcal{N}} \hat{\mathbf{\Psi}}_{\mathcal{N}}^{H} \mathbf{e}_{n}\right)^{-1 / 2} \hat{\mathbf{\Psi}}_{\mathcal{N}} \hat{\mathbf{\Psi}}_{\mathcal{N}}^{H} \mathbf{e}_{n}
$$

and (15) are equivalent. Thus, the robust solution presented herein (in the sense of minimum inter-system interference) enjoys the attractive properties of linear predictors, as minimum norm does [31]. Notice that, although all columns of $\hat{\mathbf{P}}_{\mathcal{N}} \triangleq \hat{\boldsymbol{\Psi}}_{\mathcal{N}} \hat{\mathbf{\Psi}}_{\mathcal{N}}^{H}$ are orthogonal to the sensed signal subspace, minimum inter-system interference and the advantages of linear prediction can only be guaranteed when the selected column fulfills the minimum-norm criterion [22, Sec. III].

\section{A. Multi-Waveform Design Scheme}

It is worth pointing out that even though the $N$ columns of $\hat{\mathbf{P}}_{\mathcal{N}}$ are orthogonal to the sensed signal subspace $\hat{S}$, they are not orthogonal between them. This follows from noting that $\hat{K}<N$. Therefore, the proposed solution is not feasible for multi-carrier systems. In the sequel, we present an iterative algorithm to obtain a set of $\hat{K}$ TLS orthogonal waveforms, namely $\mathcal{W}$, at an arbitrary geographical position. We assume that the internal node has detected both signal and noise subspace bases $\hat{\Psi}_{\mathcal{S}}$ and $\hat{\Psi}_{\mathcal{N}}$. We denote the set of columns of $\hat{\mathbf{P}}_{\mathcal{N}}$ that meet the minimum-norm condition as $\mathcal{F}$, with $|\mathcal{F}|=J<N$. One of them is arbitrarily selected with probability $\frac{1}{J}$. The selected solution $\varphi_{n}^{\star}(1)$ is the waveform designed at iteration $\iota=1$. For $\iota=2$, internal node should consider a modified orthogonal projector, such that it belongs to a subspace orthogonal to $\hat{\mathcal{S}} \cup\left\{\varphi_{n}^{\star}(1)\right\}$. Hence, this orthogonal projector is given by

$$
\hat{\mathbf{P}}_{\mathcal{N}}(2)=\mathbf{I}_{N}-\left[\hat{\boldsymbol{\Psi}}_{\mathcal{S}} \hat{\boldsymbol{\Psi}}_{\mathcal{S}}^{H}+\boldsymbol{\varphi}_{n}^{\star}(1)\left(\boldsymbol{\varphi}_{n}^{\star}(1)\right)^{H}\right],
$$

where $\hat{\mathbf{P}}_{\mathcal{S}}(2)=\hat{\boldsymbol{\Psi}}_{\mathcal{S}} \hat{\mathbf{\Psi}}_{\mathcal{S}}^{H}+\boldsymbol{\varphi}_{n}^{\star}(1)\left(\boldsymbol{\varphi}_{n}^{\star}(1)\right)^{H}$ is the orthogonal projector onto the modified signal subspace, i.e. the subspace encompassing the sensed signal subspace and the selected waveform at the previous iteration. Therefore, by arbitrarily selecting an element from $\mathcal{F}(2)$, internal node obtains a waveform orthogonal to both $\hat{\mathcal{S}}$ and $\varphi_{n}^{\star}(1)$. By iterating this 


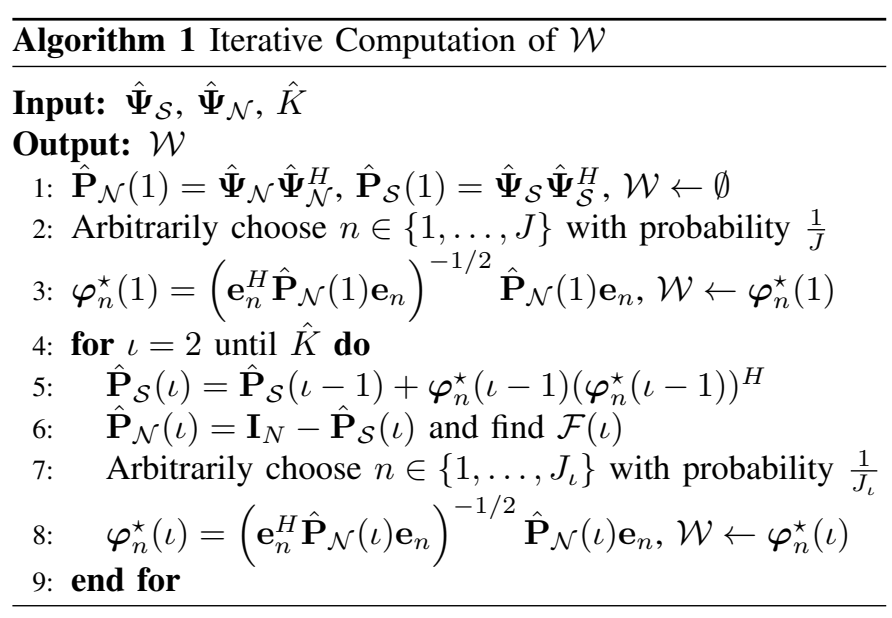

methodology until $\iota=\hat{K}$, the set $\mathcal{W}$ can be designed. The whole procedure is sketched in Algorithm 1.

\section{Properties of Robust Context-Aware Signaling}

In this Section, we highlight some properties of derived solution. Notice that a more accurate analysis was performed by the authors in [22-24].

\section{A. Invariance to Subspace Rotations}

In contrast to other works based on null-space of the external network, the waveform design scheme proposed herein relies on the orthogonal projector onto this subspace and not on a basis. Therefore, by letting $\mathbf{U} \in \mathbb{C}^{\hat{K} \times \hat{K}}$ be a unitary matrix, it is straightforward to see that

$$
\hat{\mathbf{P}}_{\mathcal{N}}^{(r)}=\hat{\mathbf{\Psi}}_{\mathcal{N}}^{(r)}\left(\hat{\mathbf{\Psi}}_{\mathcal{N}}^{(r)}\right)^{H}=\hat{\mathbf{\Psi}}_{\mathcal{N}} \mathbf{U} \mathbf{U}^{H} \hat{\mathbf{\Psi}}_{\mathcal{N}}^{H}=\hat{\mathbf{P}}_{\mathcal{N}}
$$

Hence, since the orthogonal projector is not affected by this rotation, TLS waveforms exhibit rotational invariance, which guarantees coherent detection.

\section{B. Invariance to Phase and/or Frequency Errors}

Waveform design schemes based on observations from external network acquired by a centralized unit may suffer from reference error. Nevertheless, since the derived waveforms rely on the local orthogonal projector, and defining $\Gamma$ as

$$
\boldsymbol{\Gamma}=\operatorname{diag}\left(\exp \left\{j 2 \pi v_{0}+\phi_{0}\right\}, \ldots, \exp \left\{j 2 \pi N v_{0}+\phi_{0}\right\}\right),
$$

i.e. a diagonal matrix containing the reference errors, we may observe that orthogonal projector is not modified by $\boldsymbol{\Gamma}$. Hence, TLS waveforms (15) is not affected by these reference errors.

\section{Robustness to Subspace Uncertainties}

This property is of paramount importance to guarantee the detectability by a neighboring node. Recall that each internalnetwork node only considers local external-network observations. Thus, noise subspaces sensed at each opportunistic system end, $\hat{\mathcal{N}}^{K_{\mathrm{T}}}$ and $\hat{\mathcal{N}}^{K_{\mathrm{R}}}$, are, in general, different. Actually, at an arbitrary geographical position we may define the noise subspace as

$$
\hat{\mathcal{N}}(\mathbf{r})=\mathcal{N}_{0} \cup \mathcal{E}(\mathbf{r}),
$$

where $\mathcal{N}_{0}$ encompasses the DoF that have been simultaneously detected as available by both transmitter and receiver, whereas $\mathcal{E}(\mathbf{r})$ contains those DoF only sensed as available at position r. As proven in [24], the orthogonal projector onto the sensed noise subspace at position $\mathbf{r}$ can be written as

$$
\hat{\mathbf{P}}_{\mathcal{N}}(\mathbf{r})=\hat{\mathbf{P}}_{\mathcal{N}_{0}}+\hat{\mathbf{P}}_{\mathcal{E}(\mathbf{r})},
$$

and the matched-filter miss-matching factor between the waveforms designed at the transmitter and the receiver, $\varphi_{n, \mathrm{~T}}^{\star}$ and $\varphi_{n, \mathrm{R}}^{\star}$, yields

$$
\left(\boldsymbol{\varphi}_{n, \mathrm{R}}^{\star}\right)^{H} \boldsymbol{\varphi}_{n, \mathrm{~T}}^{\star}=\left(1+\frac{\kappa_{\mathrm{T}}+\kappa_{\mathrm{R}}}{K_{0}}+\frac{\kappa_{\mathrm{T}} \kappa_{\mathrm{R}}}{K_{0}^{2}}\right)^{-1 / 2},
$$

being $K_{0}=\operatorname{dim}\left(\mathcal{N}_{0}\right)$, and $\kappa_{\mathrm{T}}=\operatorname{dim}\left(\mathcal{E}\left(\mathbf{r}_{\mathrm{T}}\right)\right)$ and $\kappa_{\mathrm{R}}=$ $\operatorname{dim}\left(\mathcal{E}\left(\mathbf{r}_{\mathrm{R}}\right)\right)$, where $\mathbf{r}_{\mathrm{T}}$ and $\mathbf{r}_{\mathrm{R}}$ refer to the positions of internal transmitting and receiving nodes, respectively. Equation (24) means that the energy injected/sensed in $\mathcal{N}_{0}$ will be preserved, whereas the fraction of energy injected/sensed in $\mathcal{E}(\mathbf{r})$ introduces a performance worsening in the form of energy loss (at transmitter) and noise enhancement (at receiver).

\section{Distribution of Waveform's Zeros}

It is worth noting that TLS and minimum-norm waveforms presented in [22-24] are equivalent. As presented by Kumaresan in [32], the so-called extraneous zeros present an almost uniform distribution inside the unit circle (and only will occupy the unit circle whenever the waveforms are linear combination of complex exponentials). Therefore, it is worth noting that proposed waveforms exhibit minimum phase and minimum group delay. Furthermore, as an additional interpretation, this (almost) uniform distribution indicates that transmitted power will be spread over all DoF detected as available. Thus, in case of sensing errors, the interference power is minimized.

\section{NumericAl ANALYSis}

In this Section, we report a numerical analysis of the proposed waveform design. For the sake of concreteness, we are going to consider that sensed signal/noise subspace bases are Fourier matrices. This case corresponds to an OFDMA scenario. Nevertheless, the results can be extrapolated to any arbitrary bases.

\section{A. Behavior Assessment: Proof-of-Concept}

We consider a wireless environment composed of $M$ heterogeneous nodes, exploiting $D$ DoF (carriers) of a 32dimensional ambient space. In this proof-of-concept, we assume a DoF occupation of $D / N=3 / 8$. Therefore, a total of $K=20 \mathrm{DoF}$ are available for opportunistic transmission.

In order to take sensing uncertainties into account, we are going to use the concept of normalized uncertainty. By letting $\xi$ be the number of occupied DoF erroneously sensed as available, the normalized uncertainty is defined as $u=\xi / D$, such that $u \in[0,1]$. It is worth noting that $u$ indicates the relative excess of DoF sensed as available. We have depicted the power spectral density (PSD) and zeros distribution in Figs. 3 and 4 , for the extreme cases $u=0$ and $u=1$. It is worth noting that the transmitted power is spread among 


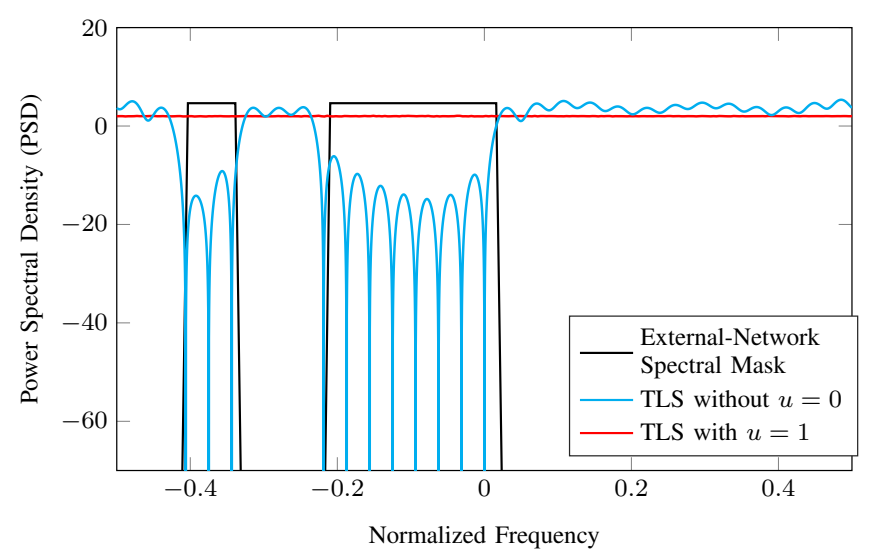

Fig. 3: PSD of TLS waveform (15) in a proof-of-concept (useless) case with a very low subspace dimension $(N=32 \mathrm{DoF})$.

all available DoF (carriers). When $u=0$, the sensed noise subspace perfectly match with the available DoF. Contrary, when $u=1$, all DoF are sensed as available and hence the transmitted power is spread over all system bandwidth. By observing Fig. 4, we see that zeros corresponding to occupied DoF are located at specific positions, whereas noise-subspace zeros are uniformly distributed inside the unit circle. From Figs. 3 and 4 follows that proposed waveform presents a behavior similar to multi-carrier CDMA.

Summarizing, notice that power is actually spread among all available subspace. For that reason, as discussed by the authors in [22], proposed transmission scheme can be seen as a dimension-based spreading mechanism. A very engaging consequence of the latter is that transmitted power per DoF (carrier) is, in average, $S_{k}=S_{\mathrm{T}} / \hat{K}$, for $k=1, \ldots, \hat{K}$, with $S_{\mathrm{T}}$ and $\hat{K}$ representing the transmitted power and the DoF sensed as available, respectively. Therefore, in case of sensing uncertainties (i.e. $u \neq 0$ ), the power delivered to those DoF erroneously sensed as available is the same as the remaining ones. Interestingly, when $S_{\mathrm{T}}$ is kept constant, the transmitted power per DoF decreases with $\hat{K}$ (i.e. decreases as the uncertainty grows). Hence, the average inter-system interference per DoF decreases with $u$.

As a second interpretation, it is worth noting that spread spectrum has found to be the optimal solution in noncooperative communications when one or more nodes present a selfish behavior and interfering systems are also spreading their transmissions in varying environments [33], such as heterogeneous networks. Hence, proposed dimensions spreading mechanism also seems to be robust to these unfair behaviors.

\section{B. Multi-Waveform Solution}

As an extension of the proposed TLS waveform, we have also proposed a multi-waveform solution. Thanks to the presented iterative algorithm, internal-network nodes can construct a waveform-book of $\hat{K}(\mathbf{r})$ elements. This procedure is very useful since enables TLS waveform design scheme in multi-carrier like scenarios and also in multi-user opportunistic communications. In order to illustrate the performance of Algorithm 1, we have depicted in Fig. 5 the three first elements

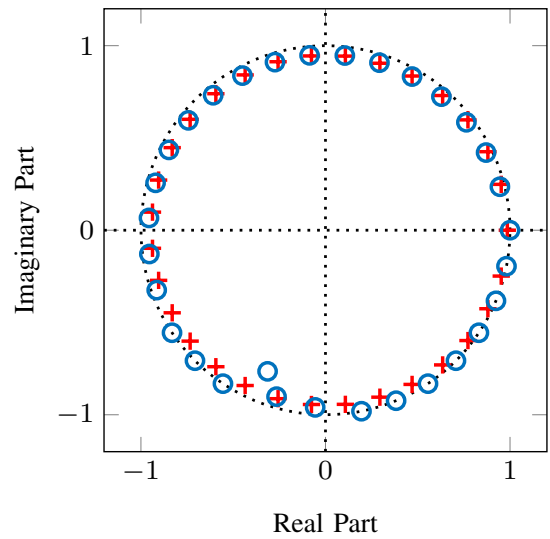

Fig. 4: Zeros distribution of proposed waveform (15) when $u=0$ (blue circles) and $u=1$ (red crosses).

of the waveform-book at geographical position $\mathbf{r}, \mathcal{W}(\mathbf{r})$. To account for a more realistic scenario than in the previous proof-of-concept, we have considered an ambient space of $N=512$ DoF. However, we have also set an external-network occupation of $3 / 8$. By observing Fig. 5 , we may see that the three waveforms depicted only span the transmitted power over the available frequency bands, keeping the external-network spectral mask unaffected (whenever $u=0$ ). What is very interesting is that thanks to the proposed iterative algorithm, all waveforms in $\mathcal{W}(\mathbf{r})$ will not only be orthogonal to the externalnetwork signal subspace, but also between them. Therefore, proposed multi-waveform transmission scheme will also avoid any kind of adjacent-waveform interference and will produce a little or non existent interference to external-network users (depending on the sensing uncertainty $u$ ).

\section{Performance of Opportunistic Transmission}

Even though sensing uncertainties may not degrade externalnetwork performance, a degradation in opportunistic transmission is observed. Recalling (24), the matched-filter gain is directly affected by subspace uncertainties at internal transmitting and receiving nodes. For the sake of illustration, the contour plot of the matched-filter gain is depicted in Fig. 6 for different normalized DoF excess $\left(\rho_{\mathrm{i}}=\kappa_{\mathrm{i}} / K_{0}\right.$, for $\left.\mathrm{i}=\{\mathrm{T}, \mathrm{R}\}\right)$ at internal transmitter and receiver. Notice that for uncertaintylimited regimes, performance of opportunistic communications might be severely degraded. Opportunistic system performance can be improved by means of subspace agreement [34].

\section{CONCLUSIONS}

This paper has dealt with the robust scenario-aware waveform design in the context of opportunistic communication. We have proven that, when the problem is cast as a total leastsquares optimization, the robustness against sensing uncertainties is guaranteed. Since proposed waveform design scheme relies on the orthogonal projector, a set of orthogonal waveform can be iteratively construct making our proposal feasible in multi-carrier systems. Moreover, total least-squares waveforms exhibits invariance to rotations, reference errors and subspace uncertainties, and their zeros are almost uniformly distributed inside the unit circle. 


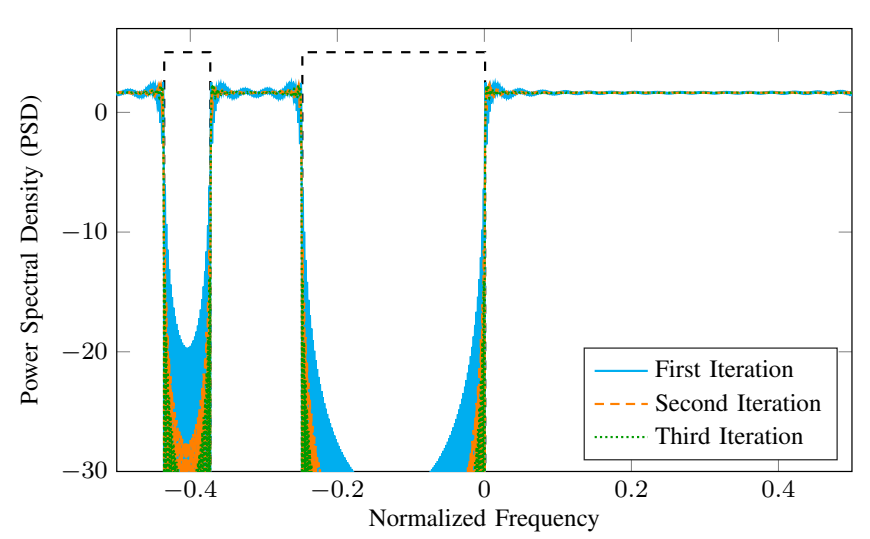

Fig. 5: PSD of the three first waveforms produced by Algorithm 1 for the external-network spectral mask depicted in dashed black.

\section{REFERENCES}

[1] J. G. Andrews, S. Buzzi, W. Choi, S. V. Hanly, A. Lozano, A. C. K. Soong, and J. C. Zhang, "What will 5G be?" IEEE J. Sel. Areas Commun., vol. 32, no. 6, pp. 1065-1082, June 2014.

[2] N. Lee and R. W. Heath Jr, "Advanced interference management technique: potentials and limitations," IEEE Wireless Commun., vol. 23, no. 3, pp. 30-38, June 2016.

[3] L. Sun, Q. Du, P. Ren, and Y. Wang, "Two birds with one stone: Towards secure and interference-free D2D transmissions via constellation rotation," IEEE Trans. Veh. Technol., vol. 65, no. 10, pp. 8767-8774, Oct 2016.

[4] D. Darsena, G. Gelli, and F. Verde, "Joint channel estimation, interference cancellation, and data detection for ambient backscatter communications," in 2018 IEEE 19th Int. Workshop Signal Process. Adv. Wireless Commun. (SPAWC), June 2018, pp. 1-5.

[5] L. Lu, G. Y. Li, A. Maaref, and R. Yao, "Opportunistic transmission exploiting frequency- and spatial-domain degrees of freedom," IEEE Wireless Commun., vol. 21, no. 2, pp. 91-97, April 2014.

[6] R. Marin, R. Ciobanu, and C. Dobre, "Improving opportunistic networks by leveraging device-to-device communication," IEEE Commun. Mag., vol. 55, no. 11, pp. 86-91, Nov 2017.

[7] D. Lopez-Perez, I. Guvenc, G. de la Roche, M. Kountouris, T. Q. S. Quek, and J. Zhang, "Enhanced intercell interference coordination challenges in heterogeneous networks," IEEE Wireless Commun., vol. 18, no. 3, pp. 22-30, June 2011.

[8] A. Goldsmith, S. A. Jafar, I. Maric, and S. Srinivasa, "Breaking spectrum gridlock with cognitive radios: an information theoretic perspective," Proc. IEEE, vol. 97, no. 5, pp. 894-914, May 2009.

[9] F. Jameel, Z. Hamid, F. Jabeen, S. Zeadally, and M. A. Javed, "A survey of device-to-device communications: research issues and challenges," IEEE Commun. Surveys Tut., pp. 1-1, 2018.

[10] Y. Zhang, E. DallAnese, and G. B. Giannakis, "Distributed optimal beamformers for cognitive radios robust to channel uncertainties," IEEE Trans. Signal Process., vol. 60, no. 12, pp. 6495-6508, Dec 2012.

[11] L. S. Cardoso, M. Kobayashi, F. R. P. Cavalcanti, and M. Debbah, "Vandermonde-subspace frequency division multiplexing for two-tiered cognitive radio networks," IEEE Trans. Commun., vol. 61, no. 6, pp. 2212-2220, June 2013.

[12] L. Lu, G. Y. Li, and A. Maaref, "Spatial-frequency signal alignment for opportunistic transmission," IEEE Trans. Signal Process., vol. 62, no. 6, pp. 1561-1575, March 2014.

[13] M. Bica, K. Huang, U. Mitra, and V. Koivunen, "Opportunistic radar waveform design in joint radar and cellular communication systems," in 2015 IEEE Global Commun. Conf. (GLOBECOM), Dec 2015, pp. 1-7.

[14] W. Hedhly, O. Amin, and M. Alouini, "Interweave cognitive radio with improper Gaussian signaling," in 2017 IEEE Global Commun. Conf. (GLOBECOM), Dec 2017, pp. 1-6.

[15] F. Zhou, Y. Wu, Y. Liang, Z. Li, Y. Wang, and K. Wong, "State of the art, taxonomy, and open issues on cognitive radio networks with NOMA," IEEE Wireless Commun., vol. 25, no. 2, pp. 100-108, April 2018.

[16] D. Ramirez, L. Huang, Y. Wang, and B. Aazhang, "On opportunistic mmWave networks with blockage," IEEE J. Sel. Areas Commun., vol. 35, no. 9, pp. 2137-2147, Sep. 2017.

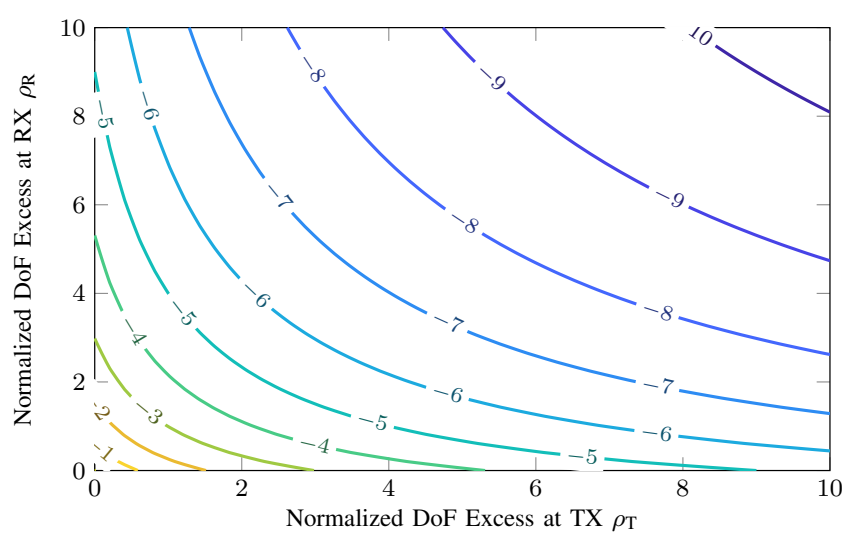

Fig. 6: Contour plot of energy losses $[\mathrm{dB}]$ as a function of normalized subspace dimension excesses at internal transmitter and receiver, $\rho_{\mathrm{T}}$ and $\rho_{\mathrm{R}}$.

[17] S. K. Sharma, T. E. Bogale, L. B. Le, S. Chatzinotas, X. Wang, and B. Ottersten, "Dynamic spectrum sharing in $5 \mathrm{G}$ wireless networks with full-duplex technology: Recent advances and research challenges," IEEE Commun. Surveys Tut., vol. 20, no. 1, pp. 674-707, Firstquarter 2018.

[18] Z. Chen and M. Kountouris, "Decentralized opportunistic access for D2D underlaid cellular networks," IEEE Trans. Commun., vol. 66, no. 10, pp. 4842-4853, Oct 2018.

[19] S. Akin and M. C. Gursoy, "Performance analysis of cognitive radio systems with imperfect channel sensing and estimation," IEEE Trans. Commun., vol. 63, no. 5, pp. 1554-1566, May 2015.

[20] A. Patel, M. Z. A. Khan, S. N. Merchant, U. B. Desai, and L. Hanzo, "The achievable rate of interweave cognitive radio in the face of sensing errors," IEEE Access, vol. 5, pp. 8579-8605, 2017.

[21] H. Lin and W. Shin, "Multi-cell aware opportunistic random access," in 2017 IEEE Int. Symp. Inf. Theory (ISIT), June 2017, pp. 2533-2537.

[22] J. Borràs, J. Font-Segura, J. Riba, and G. Vazquez, "Dimension spreading for coherent opportunistic communications," in 2017 51st Asilomar Conf. Signals, Syst. Comput., Oct 2017, pp. 1940-1944.

[23] J. Borràs and G. Vazquez, "Uncoordinated space-frequency pilot design for multi-antenna wideband opportunistic communications," in 2018 IEEE 19th Int. Workshop Signal Process. Adv. Wireless Commun. (SPAWC), Jun 2018, pp. 1-5.

[24] J. Borràs and G. Vazquez, "Decentralized shaping for pilot generation and detection in opportunistic communications," in 2019 IEEE Int. Conf. Commun. (ICC), May 2019, pp. 1-6.

[25] R. G. Gallager, Information Theory and Reliable Communications. John Wiley \& Sons, 1968.

[26] D. Tse and P. Viswanath, Fundamentals of Wireless Communication. Cambridge University Press, 2005.

[27] J. Font-Segura, G. Vázquez, and J. Riba, "Single and multi-frequency wideband spectrum sensing with side-information," IET Signal Process., vol. 8, no. 8, pp. 831-843, 2014.

[28] F. Awin, E. Abdel-Raheem, and K. Tepe, "Blind spectrum sensing approaches for interweaved cognitive radio system: A tutorial and short course," IEEE Commun. Surveys Tut., vol. 21, no. 1, pp. 238-259, Firstquarter 2019

[29] I. Markovsky and S. Van Huffel, "Overview of total least-squares methods," Signal Process., vol. 87, no. 10, pp. 2283-2302, 2007.

[30] E. M. Dowling and R. D. DeGroat, "The equivalence of the total least squares and minimum norm methods," IEEE Trans. Signal Process., vol. 39, no. 8, pp. 1891-1892, Aug 1991.

[31] D. W. Tufts and R. Kumaresan, "Estimation of frequencies of multiple sinusoids: making linear prediction perform like maximum likelihood," Proc. IEEE, vol. 70, no. 9, pp. 975-989, Sept 1982.

[32] R. Kumaresan, "On the zeros of the linear prediction-error filter for deterministic signals," IEEE Trans. Acoust., Speech, Signal Process., vol. 31, no. 1, pp. 217-220, February 1983.

[33] R. Etkin, A. Parekh, and D. Tse, "Spectrum sharing for unlicensed bands," IEEE J. Sel. Areas Commun., vol. 25, no. 3, pp. 517-528, April 2007.

[34] J. Borràs and G. Vazquez, "Distributed feedback-aided subspace concurrent opportunistic communications," in 2019 IEEE 20th Int. Workshop Signal Process. Adv. Wireless Commun. (SPAWC), July 2019, pp. 1-5. 